\title{
Sensory-Specific and Motivation-Specific Satiety for the Sight and Taste of Food and Water in Man
}

\author{
EDMUND T. ROLLS, BARBARA J. ROLLS AND EDWARD A. ROWE \\ Department of Experimental Psychology, University of Oxford, South Parks Road, Oxford OX1 3UD, England
}

Received 14 August 1982

\begin{abstract}
ROLLS, E. T., B. J. ROLLS AND E. A. ROWE. Sensory-specific and motivation-specific satiety for the sight and taste of food and water in man. PHYSIOL BEHAV 30(2) 185-192, 1983.-In normal weight humans it was shown first that the sight of food is pleasant when hungry, and that the pleasantness of the sight of a food which has been eaten to satiety decreases more than the pleasantness of the sight of foods which have not been eaten. Thus the specificity of satiety extends to the visual modality, and this may be an important factor in influencing which foods are selected for ingestion. Second, it was shown that the pleasantness of both the sight and taste of food are modulated in a motivation-specific manner, in that in hungry and thirsty humans the pleasantness of the sight and taste of food but not water is decreased by eating to satiety, and the pleasantness of the sight and taste of water but not food is decreased by drinking water to satiety. Third, it was shown that sensory-specific decreases in the pleasantness of the taste of a particular food produced by its ingestion are associated with only minor changes in the intensity of its taste, which do not account for the changes in pleasantness as shown by an analysis of covariance and by the relation between pleasantness and intensity, so that it is unlikely that sensory adaptation is an important part of the mechanism of sensory-specific satiety.
\end{abstract}

Eating Drinking Sensory-specific satiety Human feeding Motivation Taste Food preference Meal size

DURING investigations of the neural controls of feeding, neurons have been found in the hypothalamus of the monkey which respond to the sight and/or taste of food [16-24]. The responsiveness of these neurons is modulated by hunger, in that their responses to food become attenuated after feeding to satiety [1]. Interestingly, though, it was found that this decrease of neuronal responsiveness was much greater for a food on which the monkey had been satiated, and that these neurons might still respond at least to some extent to other foods on which the monkey had not been satiated (observations of $E$. T. Rolls and colleagues; see $[17,18,20])$. In line with this neurophysiological indication for partial specificity of satiety for a food which has just been eaten, the monkey was then willing to accept and eat the other foods on which he had not been fed to satiety.

These neurophysiological findings led to our investigations of the degree to which satiety might be specific in man. It had previously been shown by Cabanac and his colleagues $[2,3]$ that the pleasantness of food-related odors or of the taste of sucrose was reduced by a $50 \mathrm{~g}$ load of glucose. He argued that there is modulation of the hedonic responses to food produced by the internal nutritional state which could be important in controlling feeding, and termed the phenomenon "alliesthesia" [2]. To investigate the degree to which satiety might be specific in man, we allowed subjects to eat as much of one particular food as they wished, and found that the pleasantness of the food eaten decreased more than the pleasantness of seven other foods which had not been eaten $[12,20]$. Because this phenomenon was at least partly specific for the sensory qualities of the food eaten, and was not solely dependent on metabolic feedback (in that some of the foods eaten were nutritionally similar, the effect had a short onset latency, and the effect could be obtained with low energy foods) $([12,28]$ and unpublished observations of E. T. Rolls and B. J. Rolls), it was called "sensory-specific satiety" [12], following earlier terminology of Le Magnen [5]. This sensory-specific satiety was seen not only as a relatively greater decrease in the pleasantness of the taste of a food eaten than in that of foods not eaten, but was also a factor which influenced the subsequent intake of foods. Thus, in a separate experiment it was shown that if subjects ate one food until they were satisfied and there was a sensory-specific decrease in its pleasantness, then relatively little of this food was eaten in an unexpected second course, whereas if a food not eaten in the first course which was still relatively pleasant to taste was offered in the second course, relatively much of it was eaten [12]. Further, it was shown that this sensory-specific satiety effect may be an important influence on the amount of food eaten, in that intake was greater in a meal in which variety rather than a single food was offered [13]. A similar effect of variety on meal intake has been demonstrated in the rat [27], and it has been shown in the rat that variety is sufficient to lead in the long term to a significant increase in body weight ([27] and Rolls, van Duijvenvoorde and Rowe, in preparation).

Given this previous neurophysiological work with com- 
plementary investigations in man, the aims of the research described here were to analyse further the nature of sensory-specific satiety. First, it has been shown that the sight of food is a powerful influence on some hypothalamic neurons in the primate, and that this influence shows sensory-specific satiety $[17,20,23]$. This population of neurons may be important in guiding behavior towards food in the appetitive phase of feeding. As investigations of sensory-specific satiety in man have so far been directed towards measurements of how the ingestion of food influences the taste or smell of food [12], the first aim of the present investigation was to determine whether, in correlation with the neurophysiological findings, the sight of food was pleasant during hunger, and whether it showed a partly sensory-specific decrease in pleasantness after eating a food.

Second, there is evidence for motivation-specific response systems in the primate hypothalamus. That is, some neurons respond to the sight or taste of food but not water, and other neurons respond to the sight and/or taste of water but not food $[18,23]$. Such motivational specificity is required of a system which is to guide and control hunger or thirst motivated behavior, for approach to and consumption of food is appropriate when hungry, and of water when thirsty. A second aim of the investigations described here was then to determine whether similarly the pleasantness of food but not water is modulated by hunger, and the pleasantness of water but not food is modulated by thirst, as these are conditions for pleasantness having a role in guiding specifically either feeding or drinking.

A third aim of the present investigation was to provide evidence on the mechanism of sensory-specific satiety by determining whether when there was a sensory-specific decrease in the pleasantness of a food, the intensity or strength of its taste also diminished. If its intensity did decrease, this would suggest that sensory adaptation might play a role in sensory-specific decreases in pleasantness. If on the other hand, the pleasantness decreased as a result of feeding, but the intensity remained relatively unchanged, this would suggest that adaptation early in the sensory pathways does not account for sensory-specific satiety, but rather that sensory-specific satiety involves a change in a mechanism concerned particularly with the reward or hedonic value of food. This latter mechanism might be more adaptive in that otherwise it might not be possible to taste a food after it had been eaten to satiety, and might be more in line with observation, in that subjects still taste food after eating it, but it no longer tastes as pleasant. There is little previous experimental evidence on this. Mower et al. [7] found that the perceived intensity of food-related olfactory and gustatory stimuli was not affected by internal state, that is by whether the subjects were fasted, were not fasted, or had been given a glucose load.

These three aims were incorporated into the design of the first experiment described here. Human subjects rated the pleasantness of the sight and taste, and the intensity of the taste, of several foods and of water while they were both hungry and thirsty. Then on 3 different days they ate one food, a different food, or drank water, and then performed the ratings again. Comparisons of the changes in ratings after ingestion of the 2 different foods allowed sensory-specific effects for feeding to be measured, and comparisons of the changes after ingestion of food as opposed to water allowed motivation-specific effects to be measured. The second set of ratings was taken immediately after the subjects had eaten to satiety, as we wished to investigate the factors which nor- mally influence whether food is eaten or not, and these should be evident from the pre-to post-feeding comparison. Orange flavored water was included in the taste substances in order to assess whether any change of the rating of water after ingestion of water generalized more to other rehydrating substances than to foods, that is in accordance with a motivation-specific mechanism, rather than just a sensoryspecific mechanism. Real foods were used, to make the results as relevant as possible to the normal control of human food intake. The scale on which intensity was rated was of a similar type, namely a visual analog scale, to that used for the pleasantness ratings, so that possible differences between the changes of intensity and pleasantness could not be attributed simply to gross differences in the type of rating scale used

\section{EXPERIMENT 1}

\section{METHOD}

Twenty-four subjects, 12 male and 12 female, were selected from a subject pool of healthy young adults. Subjects receiving medication, smokers, dieters, and those who disliked any of the types of food used in the experiments were excluded in an initial screening test, and care was taken to exclude subjects who had prior knowledge of this type of experiment (Psychology students were not used). The subject's body weights and heights were measured and a body mass index (Quetelet index, weight in $\mathrm{kg} /\left(\right.$ height in $\mathrm{m}^{2}{ }^{2}$ ) was calculated for each subject [4]. No subject was obese according to this index (range of body mass index, men 19.2-23.5, women 16.6-24.0), and only one female subject had a greater body weight relative to height and frame size than the desirable level given in the Metropolitan Life Insurance Tables [6]. The subjects came to the laboratory hungry and thirsty at 1 p.m. for lunch, having taken a normal breakfast, except that only one cup of fluid was allowed, and having consumed nothing since breakfast. They recorded what they ate for breakfast, and ate a similar breakfast on each of the experimental days, which were separated by at least 1 day.

On arrival at the laboratory, hunger and thirst were assessed using visual analog rating scales. These scales were $100 \mathrm{~mm}$ lines marked at one end "not hungry" and at the other end "extremely hungry," or marked "not thirsty" and "extremely thirsty." The subjects were asked to mark the line at the point which represented their hunger or thirst. We have used these visual analog rating scales previously, and have obtained consistent and reliable effects with them [14]. Each rating scale was on a separate slip of paper to prevent comparisons with previous ratings. The subjects then rated the pleasantness of the sight of five test substances, which were orange squash (artifically sweetened low energy orange drink, $0.14 \mathrm{~kJ} / \mathrm{g}$ ), cheese (mild Cheddar, $20 \mathrm{~kJ} / \mathrm{g}$ ) on cracker (Crawford's Cheddars, $22 \mathrm{~kJ} / \mathrm{g}$ ), sausage (English pork sausage, $18 \mathrm{~kJ} / \mathrm{g}$, which is relatively unseasoned, and high$14 \%$-in protein), water, and Smarties (Rowntree Mackintosh, colored sugar-coated chocolate similar to $\mathbf{M}$ and $\mathbf{M}$ 's in America). The ratings were always made in the order given above. The visual analog rating scale slips were headed "how pleasant does the substance look?" and the line was marked at one end "very unpleasant" and at the other "very pleasant." Then the subjects took in the above order a small sample (approximately $10 \mathrm{~g}$ ) of each substance into the mouth, rated the pleasantness and intensity of the taste of each substance for the $10 \mathrm{~s}$ period while it was in the mouth, and then swallowed it. Each rating slip was headed "How 


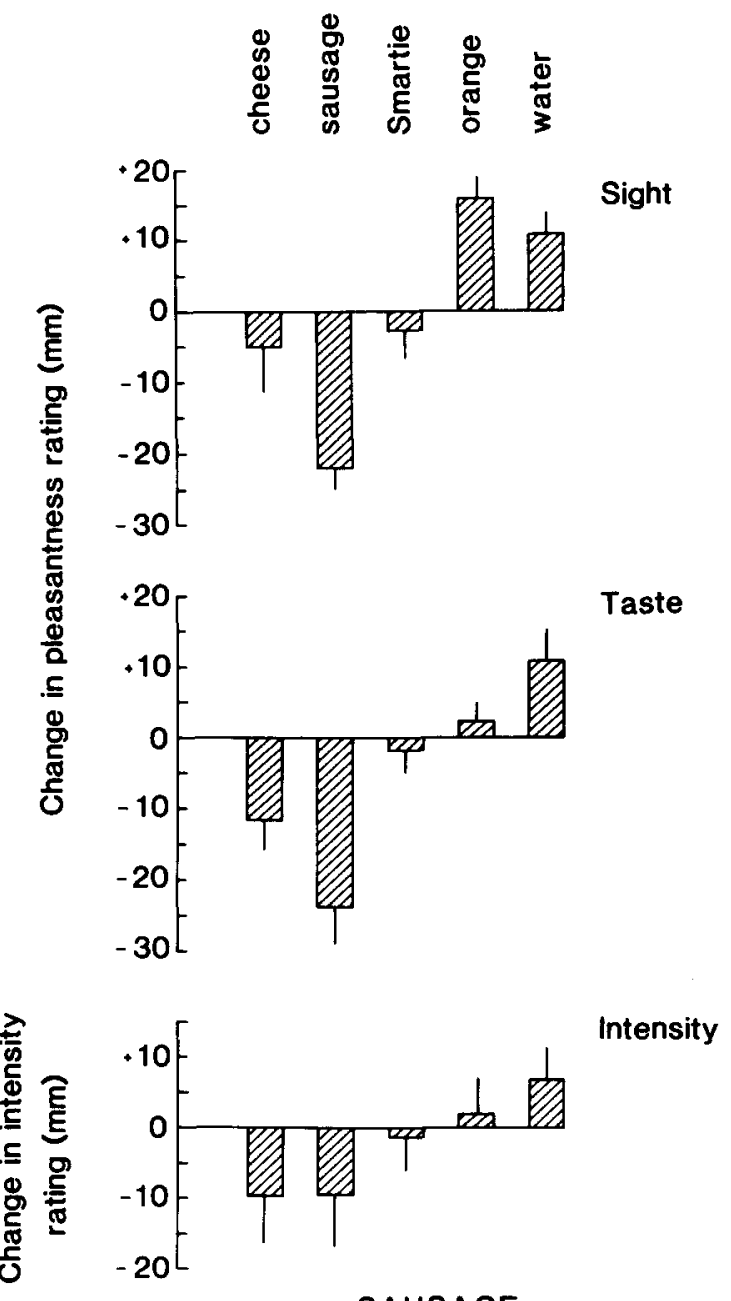

SAUSAGE

FIG. 1. The mean changes in ratings ( \pm SEM), expressed as $\mathrm{mm}$ difference on the visual analog scale, of the pleasantness of the sight (top), the pleasantness of the taste (middle), and of the intensity of the taste (bottom), of different foods and fluids produced by eating sausage. The substances rated are indicated at the top of the figure.

pleasant is the taste of the substance?" and marked "very unpleasant" and "very pleasant," or "How intense is the taste of the substance?" with "very weak" and "very intense" at the two ends of the line.

Each subject then ingested enough of either cheese on cracker, sausage, or water to satisfy him completely, and a record was kept of the weight ingested. Each subject was tested on three different experimental days, with a different substance ingested each day. The order of ingestion was counterbalanced across both male and female subjects. Within two minutes of the end of the meal, the subjects rerated the pleasantness of the sight of the 5 test substances, and then rerated the pleasantness and intensity of the taste of the test substances. They then rerated their hunger and thirst. On the last test day the subjects completed a questionnaire to determine whether they were restrained or unrestrained eaters [25], and according to this evaluation three of the subjects were mildly restrained eaters.

The changes in pleasantness, intensity, hunger and thirst,

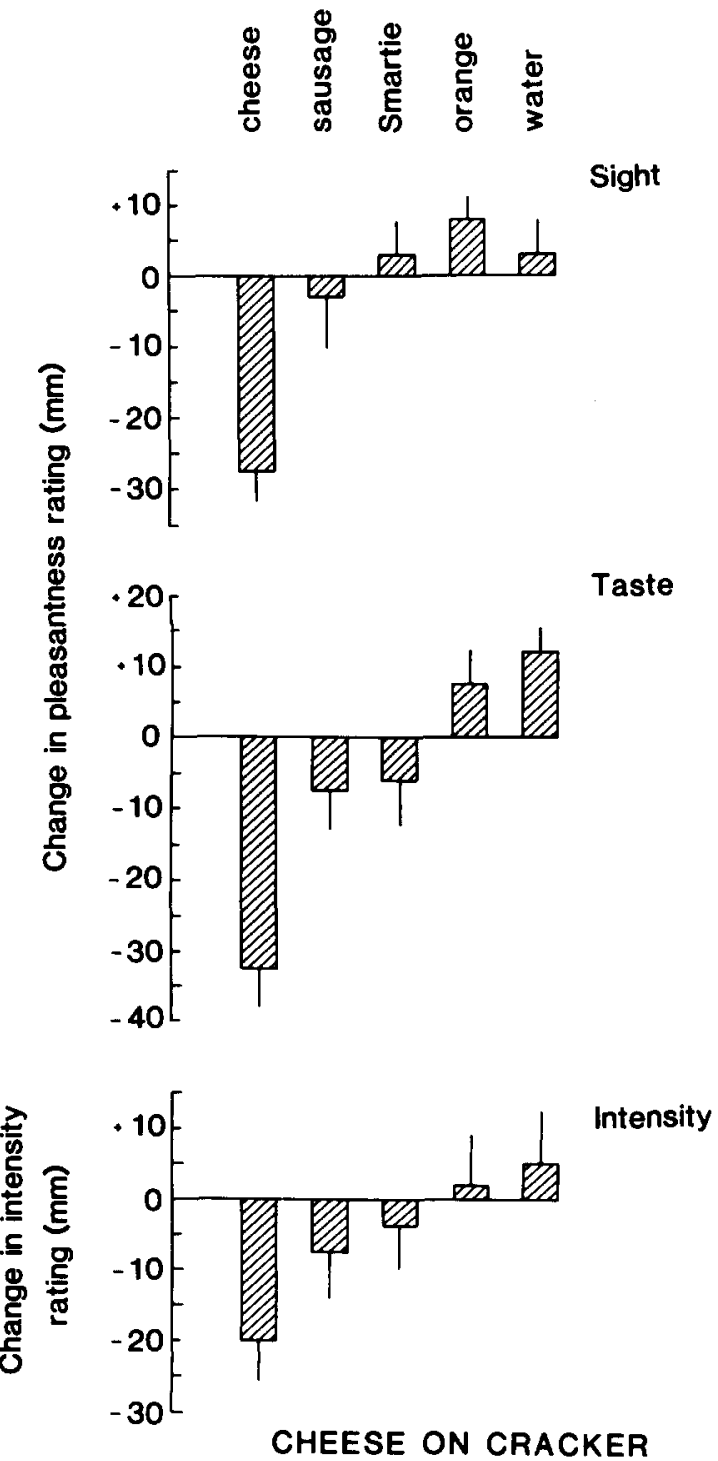

FIG. 2. The mean changes in ratings ( \pm SEM), expressed as $\mathrm{mm}$ difference on the visual analog scale, of the pleasantness of the sight (top), the pleasantness of the taste (middle), and of the intensity of the taste (bottom), of different foods and fluids produced by eating cheese on cracker.

from before eating to after eating, measured as differences in $\mathrm{mm}$ along the visual analog rating scales, were analyzed for the three different ingestion conditions with within-subjects analyses of variance followed by Newman-Keuls' analyses to locate the significant changes. The mean changes for the different conditions with their standard errors were also calculated for graphical presentation of the results. The influence of changes in intensity on the changes in pleasantness of taste was examined, where appropriate, by an analysis of covariance of the changes in pleasantness of taste by adjustment using regression analysis with the changes in intensity.

\section{RESULTS}

The results, expressed as changes in the pleasantness and intensity ratings expressed in $\mathrm{mm}$ along the visual analog 


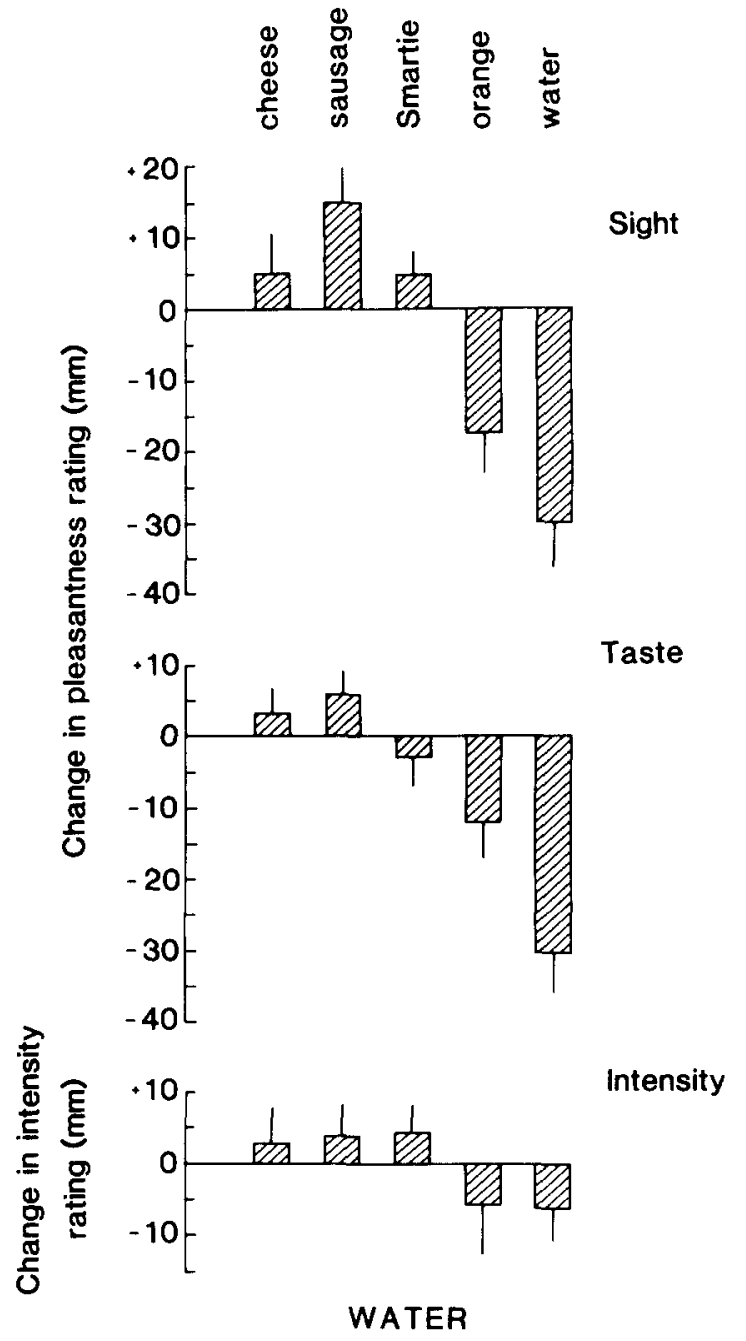

FIG. 3. The mean changes in ratings $( \pm$ SEM), expressed as $\mathrm{mm}$ difference on the visual analog scale, of the pleasantness of the sight (top), the pleasantness of the taste (middle), and of the intensity of the taste (bottom), of different foods and fluids produced by drinking water.

rating scales are shown for the three different ingestion conditions in Figs. 1-3. A negative change on the diagrams indicates that the substance became less pleasant or intense following the ingestion.

First, sensory-specific decreases in the pleasantness of the sight of a particular food which had been ingested were found. Thus after eating sausage, the pleasantness of the sight of sausage decreased more than that of each of the other four test substances (see Fig. 1) $(p<0.01$ for each comparison using a Newman-Keuls' test following a significant overall effect in the analysis of variance for the sausage ingested/sight rating condition, $\mathbf{F}(4,92)=21.2, p<0.001$ ). Similarly, after eating cheese, the pleasantness of the sight of cheese decreased more than that of each of the other 4 test substances (see Fig. 2) $(p<0.01$ for each comparison using a Newman-Keuls' test following a significant overall effect in the analysis of variance for the cheese ingested/sight rating condition, $F(4,92)=19.3, p<0.001$ ). A similar effect was found for water. Thus after drinking water, the pleasantness of the sight of water decreased more than that of each of the other four test substances (see Fig. 3) $(p<0.0$ I for each comparison using a Newman-Keuls' test following a significant overall effect in the analysis of variance for the water ingested/sight rating condition, $\mathrm{F}(4,92)=26.6, p<0.001)$.

Second, motivation-specific decreases in the pleasantness of the taste and sight of food were found after eating food, and of water after ingesting water. For example, after eating sausage, the pleasantness of the sight and taste of the three foods (cheese, sausage and Smartie) decreased, while that of water increased. (For the sight condition, for which $F(4,92)=21.3, p<0.001$ as noted above, the Newman-Keuls' analysis showed that each of the three foods. sausage, cheese and Smartie, had decreased in pleasantness relative to the sight of water, $p<0.01$ in each case. Similarly, for the taste condition, for which $F(4.92)=20.8$, the pleasantness of the taste of each of the foods decreased more in pleasantness than the taste of water: sausage or cheese vs. water $p<0.01$. Smartie vs. water $p<0.05)$. Also, after eating cheese, the pleasantness of the sight and taste of the three foods (cheese. sausage and Smartie) usually decreased (see Fig. 2), while that of water increased. (For the sight condition, for which $F(4,92)=19.3 . p<0.001$ as noted above, the Newman-Keuls analysis showed that of the 3 foods. sausage and cheese but not Smartie decreased more in pleasantness than the sight of water, $p<0.01$ in both cases. For the taste condition. for which $F(4,92)=20.5$, the pleasantness of the taste of each of the foods decreased more than the taste of water: sausage or cheese vs. water $p<0.01$. Smartie vs. water $p<0.05)$. A comparable motivation-specific decrease in the pleasantness of the sight and taste of water but not of foods occurred after drinking water. (For the sight condition, for which $\mathrm{F}(4,92)=26.6, p<0.001$ as noted above, the Newman-Keuls analysis showed that the sight of water had decreased more in pleasantness than the sight of each of the three foods, sausage, cheese and Smartic, $p<0.01$ in each case. Similarly, for the taste condition, for which $\mathrm{F}(4.92)=7.6, p<0.001$, the taste of water decreased more in pleasantness than the taste of each of the three foods, sausage. cheese, and Smartie. $p<0.01$ in each case.)

The changes in the pleasantness of the orange-flavored water were more similar to the changes in the pleasantness of water than to the changes in the pleasantness of the three foods. Thus, after drinking water, the sight and taste of orange as well as of water became less pleasant in contrast to the foods which became more pleasant. while after eating sausage or cheese, the pleasantness of orange as well as of water increased in contrast to the foods which tended to become less pleasant (see Figs. 1-3). Most of these comparisons, as specified next, reached a satisfactory level of statistical significance, as shown by the Newman Keuls" tests which followed the analyses of variance. Thus, after eating sausage. the change in pleasantness of the sight of each of the three foods was significantly different from the change in the pleasantness of the sight of orange as well as of water (all comparisons $p<0.011$. Also after eating sausage, the changes in the pleasantness of the taste of each of the foods-sausage and cheese-were significantly different from the change in the pleasantness of orange as well as of water (all comparisons $p<0.01)$. Similarly, after eating cheese, the change in the pleasantness of the sight of both orange and water was significantly different from that of sausage and cheese $(p<0.01$ or $p<0.05$ for each comparison), and for taste, the changes for both orange and water were significantly different from those for cheese, sausage and orange $(p<0.0)$ or 
$p<0.05$ for each comparison). Also, after drinking water, the change in the pleasantness of the sight of both orange and water was significantly different from that of each of the three foods-sausage, cheese and Smarties- $(p<0.01$ for each comparison), and for taste, the changes for both orange and water were significantly different from those for cheese or sausage ( $p<0.01$ or $p<0.05$ for each comparison).

Third, some, but only relatively small, changes in the ratings of the intensity of the taste of foods were produced by ingestion (see Figs. 1-3). For example no significant intensity changes were produced by the ingestion of water (Fig. 3). The analysis of variance for the effect of eating sausage on the intensity of the tasted substances was significant, $F(4,92)=5.1, p<0.01$, and the Newman Keuls' test showed that the only significant differences between the individual taste substances were that both sausage and cheese changed relative to water. The analysis of variance for the effect of eating cheese on the intensity of the tasted substances was significant, $\mathrm{F}(4,92)=4.8, p<0.01$, and the Newman Keuls' test showed that the only significant differences between the individual taste substances were that cheese changed relative to orange $(p<0.01)$, water $(p<0.01)$, sausage $(p<0.05)$ and Smarties $(p<0.05)$. The analysis of covariance of changes in pleasantness of taste after extraction of the effect of intensity on pleasantness showed that sensory-specific changes in the intensity of foods after eating cheese were not significant factors in the sensory-specific change in the pleasantness of the taste of foods after eating cheese. Thus the analysis of covariance of the pleasantness of taste adjusted by intensity showed a significant difference in the changes in ratings of the substances tasted after eating cheese, $F(4,91)=14.8, p<0.001$; the covariance was not statistically significant, $F(1,91)=2.3$; and the covariance regression coefficient between changes in pleasantness and intensity was not significant (coefficient $\pm S E M=0.135 \pm 0.089$ ). The Newman Keuls' analysis showed that the adjusted changes in pleasantness of cheese after eating cheese were significantly greater than the changes in sausage. Smarties, orange, or water $(p<0.01)$. The motivation-specific changes noted in the analysis of variance of pleasantness were evident in the analysis of covariance: the adjusted changes in the pleasantness of sausage after eating cheese were significantly greater than for water $(p<0.01)$ or orange $(p<0.05)$, and were significantly greater for Smarties than for water $(p<0.05)$.

At the start of the experiment on the different days, the subjects' hunger and thirst ratings were very consistent, with the mean hunger and thirst both within $3 \mathrm{~mm}$ of $31 \mathrm{~mm}$ from the very hungry/very thirsty end of the rating scale on the different days. After ingesting sausage, hunger decreased by $43.1 \pm 5.3 \mathrm{~mm}$ (mean \pm SEM), and thirst by $7.9 \pm 3.6 \mathrm{~mm}$. After ingesting cheese, hunger decreased by $45.3 \pm 3.4 \mathrm{~mm}$, and thirst decreased by $1.2 \pm 3.4 \mathrm{~mm}$. After drinking water, thirst decreased by $41.2 \pm 6.2 \mathrm{~mm}$, and hunger increased by $0.92 \pm 4.5 \mathrm{~mm}$. There were thus specific motivational changes produced by the different ingestion conditions.

\section{EXPERIMENT 2}

Because some changes of the intensity of the taste of a food which had been eaten to satiety were found in Experiment 1, a second experiment was performed to investigate these changes in intensity ratings further, and the extent to which they might contribute to the sensory-specific decreases in pleasantness which were found in Experiment 1.

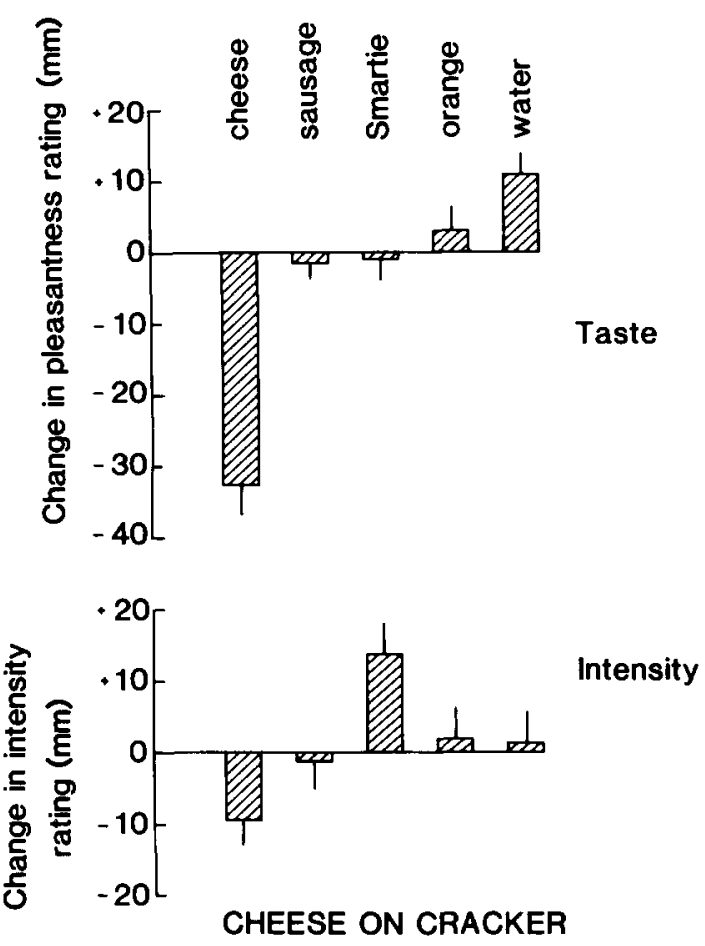

FIG. 4. The mean changes in ratings $( \pm S E M)$, expressed as $\mathrm{mm}$ difference on the visual analog scale, of the pleasantness of the taste (top), and of the intensity of the taste (bottom), of different foods and fluids produced by eating cheese on cracker in Experiment 2.

The aims of Experiment 2 were to determine how the intensity ratings were related to the concentration of the tasted substance, whether these intensity ratings were related in any simple way to the pleasantness rating or whether these were relatively independent, and whether in subjects in whom the intensity scales had been validated, similar effects to those found in Experiment 1 still occurred.

\section{METHOD}

The design of the experiment was similar to that of Experiment 1 , except that ratings of the pleasantness and intensity of a range of concentrations of sucrose and salt solution were performed first. The twenty four subjects, 12 male and 12 female, were from the same student population as in Experiment I, but were different individuals. On arrival at the laboratory, they rated the pleasantness and intensity of the taste of sucrose solutions $(0.1,0.2,0.4,0.8$, and $1.6 \mathrm{M})$ and of sodium chloride solutions $(0.1,0.2,0.4,0.8$, and $1.6 \mathrm{M}$ ) using the same type of visual analog scale as in Experiment 1. The concentrations and types of solution were tasted in a fixed randomized order, the mouth was rinsed with water between each tasting, and after one series containing each concentration once had been rated, a second series was rated. It should be noted that the subjects were not given a fixed standard, and used the $100 \mathrm{~mm}$ visual analog scales of Experiment 1 labelled at one end "very intense" or "very pleasant." and at the other end "very weak" or "very unpleasant." After performing these ratings, the subjects were tested in the condition of Experiment 1 in which cheese on cracker was ingested, except that ratings of the pleasantness of the sight of the foods were not made. 

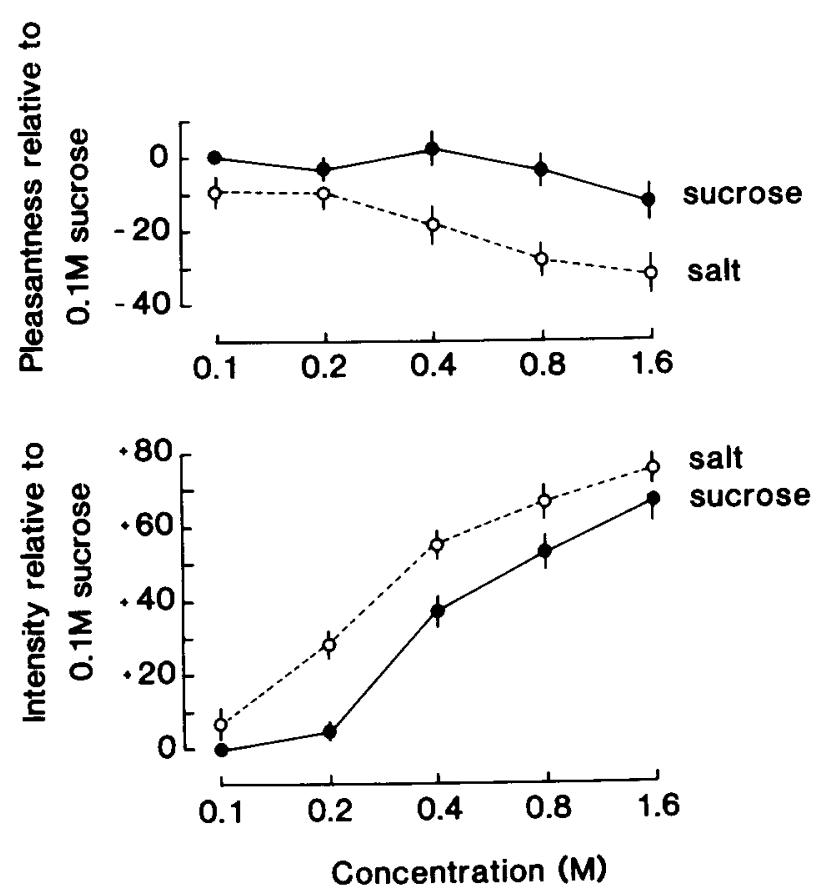

FIG. 5. The relation between the pleasantness and intensity of the taste of different concentrations of sucrose and sodium chloride solutions found in the first part of Experiment 2. The pleasantness and intensity ratings are shown as differences on the visual analog scales in $\mathrm{mm}$ from the ratings given for the pleasantness or intensity of 0.1 M sucrose solution.

\section{RESULTS}

The effects of ingestion on the changes in the pleasantness and intensity ratings are shown in Fig. 4. The effects replicated closely those obtained in Experiment 1, in that there was a sensory-specific decrease in the pleasantness of the taste of the substance ingested (cheese on cracker), and there were only relatively small changes in the intensity ratings. The analysis of variance of changes in pleasantness of taste after eating cheese showed sensory-specific changes, $\mathrm{F}(4,92)=24.3, p<0.001$, and the Newman-Keuls analysis showed that the change in pleasantness of the taste of cheese after eating cheese was significantly greater than for the changes in sausage, Smarties, orange and water $(p<0.01)$. Motivational specificity was again indicated by the finding that after eating cheese there was a greater change in sausage or Smarties than in water $(p<0.05)$. The analysis of variance of the changes in intensity of taste after eating cheese was significant, $\mathrm{F}(4,92)=4.6, p<0.01$, and this reflected significantly greater changes in the intensity of cheese $(p<0.01)$ and sausage $(p<0.05)$ than of the sweet food, Smarties. As in Experiment 1, the analysis of covariance of changes in pleasantness of taste after adjustment by the changes in intensity showed that sensory-specific changes in the intensity of foods after eating cheese were not significant factors in the sensory-specific change in the pleasantness of the taste of foods after eating cheese. Thus the analysis of covariance of pleasantness of taste adjusted by the effect of intensity on pleasantness showed a significant difference in the changes in the pleasantness of substances tasted after eating cheese, $F(4,91)=21.5$, $p<0.001$; the covariance was not statistically significant, $F(1,91)=1.6$; and the covariance regression coefficient between the changes in pleasantness and intensity was not significant (coefficient $\pm S E M=0.112 \pm 0.088$ ). The Newman Keuls' analysis showed that the adjusted changes in pleasantness of cheese after eating cheese were significantly greater than the changes in sausage, Smarties, orange or water $(p<0.01)$. The motivation-specific changes noted in the analysis of variance of pleasantness were evident in the analysis of covariance: adjusted changes in the pleasantness of the taste of the foods tasted but not eaten (Smarties and sausage) differed significantly from water $(p<0.05)$.

In the first phase of the experiment, a clear relation was found between the concentration of the solutions and the intensity ratings given by the subjects (see Fig. 5, based on the second series of ratings). For sucrose, pleasantness across the group of subjects was not related in any consistent way to concentration (see Fig. 5), with individual subjects responding with monotonic increases in pleasantness as a function of sucrose concentration, with monotonic decreases in pleasantness, with non-monotonic changes, or with no change in pleasantness. These results show that the subjects were using the intensity scale accurately, and provide an indication that it is unlikely that the change in pleasantness reported consistently across the group of subjects after ingestion of a food can be ascribed to a change in the perceived intensity of that food, as the intensity and pleasantness of different concentrations of sucrose solutions were not simply related to one another across the group of subjects. (It would be of interest to extend this type of analysis of how intensity and pleasantness are related to each other across changes in concentration of foods other than sucrose.) For saline, the intensity ratings reflected the concentration of the saline, and although the higher concentrations of saline were less pleasant, the change in pleasantness as a function of concentration was much less (in $\mathrm{mm}$ on the visual analog scale) than the change in intensity.

\section{GENERAL DISCUSSION}

These results show first that human subjective responses to the pleasantness of food can be extended to the visual modality, and that sensory-specific decreases in the pleasantness of the sight of food can be produced by eating a particular food to satiety. We were led to this experiment by the finding that in the non-human primate, the sight of food has a powerful influence on the activity of a population of hypothalamic neurons, and that the responsiveness of these neurons to food is reduced in a partly sensory-specific manner by eating a food to satiety [19, 20, 23]. These hedonic ratings in man and neurophysiological responses in the monkey underline the importance of the sight of food in the appetitive phase of food intake. Our hypothesis is that such hypothalamic neurons respond in accordance with the food reward value of the visual input, and are important in guiding behavior towards food when it is seen in the environment (see [15], pp. 21-29; see also [16-18, 23]).

Second, these results provide evidence for motivationally specific changes in the pleasantness of substances which have been ingested. We had shown previously that the pleasantness of the taste of water is decreased when thirsty humans drink water to satiety [8-10], and we and others had shown that the pleasantness of the taste of food is decreased by feeding [2.12]. Here we show that the pleasantness of the taste and of the sight of water but not of food is decreased in 
hungry and thirsty subjects by drinking water to satiety, and that the pleasantness of the taste and sight of food but not of water is decreased by eating. These effects do appear to be motivationally specific, and not just associated with sensory-specific satiety of the type found within a group of foods, in that the orange flavored drink, which differed both from the foods ingested (sausage or cheese) and from water. showed pleasantness changes which followed the pattern of changes found for water, i.e., decreasing in pleasantness after the ingestion of water and increasing in pleasantness after the ingestion of food. One reason for investigating motivational specificity is that it is required if these hedonic effects of sensory input are involved in guiding ingestion of food, which is appropriate when hungry, and of water, which is appropriate when thirsty. Another reason is that there is a neurophysiological basis for motivationally specific neural control mechanisms, in that it has been found that one population of hypothalamic neurons responds to food but not water, and a different population responds to water but not food $[18,24]$.

The difference between motivation-specific satiety and sensory-specific satiety may be emphasized clearly by considering the ingestion of a number of different foods when both hungry and thirsty. Experiments of this type have been performed on the monkey while investigating the responsiveness of hypothalamic neurons. It is found that each food produces satiety which has some specificity, but also generalizes to some extent to other foods. After eating the different foods, satiety extends to most foods, yet the appetite for water remains. In this situation in man, the water still looks and tastes pleasant. Thus the satiety for foods has motivational specificity, and does not extend to water. In the experiments described above, it was found that the pattern of subjective responses to the orange-flavored drink was similar to but not identical to that to water, and this was presumably because of a partially sensory-specific effect, in that the orange-flavored drink was not identical to water, and partly because of motivational specificity, in that the orange-flavored drink was treated more like water than food. Nor is it likely that the orange-flavored drink was treated more like water than food only because its sensory properties were more like water than food, in that, as suggested above, no range of foods, however wide, would abolish the appetite for orange-flavored water in a hungry and thirsty subject.

Third, these results show that small changes in the intensity of the taste of foods which have been ingested can occur, but suggest that these changes do not cause the changes in pleasantness which occur in sensory-specific satiety. There was for example an indication in one condition (cheese eaten) in Experiment 2 that female subjects did experience a reduction in the intensity of the food eaten. (The intensity of cheese was reduced in this condition by $20 \mathrm{~mm}$ on the visual analog rating scale, and the analysis of variance for this condition was significant, $\mathrm{F}(4,44)=5.2, p<0.001)$. There was no similar intensity change for the other food eaten by female subjects in Experiment 2, and none for either food eaten by males in Experiment 2 or for any condition in Experiment 1. Part of the evidence for the suggestion that these minor changes in intensity do not cause the changes in pleasantness which occur in sensory-specific satiety, is that no significant correlation was found between the changes in pleasantness and in intensity. Also, in subjects who had been pretrained on intensity ratings, and who had been shown to make intensity ratings which were related very well with the concentration of the sucrose or salt tasted, no simple and consistent relation between intensity and pleasantness ratings of food were found. Thus for some subjects pleasantness ratings of sucrose increased monotonically with increasing concentration. For other subjects pleasantness increased up to moderate concentrations and decreased at higher concentrations (see also [7,23]), and for other subjects the pleasantness ratings were relatively flat. The curves relating pleasantness and intensity to concentration (Fig. 5) suggest that the types of and the magnitudes of the intensity changes found after eating a food (Figs. 1 and 2) could not account for the changes in the pleasantness of the foods (Figs. 1 and 2). All these points of evidence suggest that simple sensory adaptation is not a major mechanism of sensory-specific satiety, and that the major change produced by eating a food is not in how intense it tastes, but rather in how pleasant it tastes. Further, we note that eating a particular color of food decreases the pleasantness of the taste of that food in a sensory-specific manner [14], and that in this case the change of the pleasantness of the taste cannot be due to differential adaptation of gustatory sensory pathways, as the different colored foods had the same taste. Finally, we note that how pleasant a food tastes is correlated with subsequent eating of that food [12], and thus that it is of importance to investigate the ways in which the pleasantness of food is controlled.

It was of interest that in Experiment $I$ the ratings of hunger (but not thirst) were decreased to a similar extent by eating either sausage or cheese, in contrast to the pleasantness of the sausage and cheese, which were decreased in a sensory-specific manner. This underlines the difference between hunger and appetite, where hunger reflects a predisposition to eat, but appetite reflects a predisposition to eat a particular food.

\section{ACKNOWLEDGEMENTS}

We acknowledge the support of the Medical Research Council, the assistance of Ms. S. Martin in one of the experiments, and thank United Biscuits and Rowntree Mackintosh for supplying some of the foods.

\section{REFERENCES}

1. Burton, M. J., E. T. Rolls and F. Mora. Effects of hunger on the responses of neurons in the lateral hypothalamus to the sight and taste of food. Exp Neurol 51: 668-677, 1976.

2. Cabanac, M. Physiological role of pleasure. Science 173: 11031107,1971

3. Cabanac, M. and R. Duclaux. Specificity of internal signals in producing satiety for taste stimuli. Nature 227: 966-967, 1970.
4. Cole, T. J. and W. P. T. James. The slimdicator. A slide rule device for assessing obesity. Practitioner 220: 628-629, 1978.

5. Le Magnen, J. Habits and food intake. In: Handbook of Physiology. section 6, vol. 1. Washington, DC: American Physiological Society, 1967, pp. 11-30.

6. Metropolitan Life Insurance Company. New weight standards for men and women. Stat Bull Metropol Life Insur Co 40: 1-4. 1959. 
7. Mower, G. D., R. G. Mair and T. Engen. Influence of internal factors on the perceived intensity and pleasantness of gustatory and olfactory stimuli. In: The Chemical Senses and Nutrition. edited by M. R. Kare and O. Maller. New York: Academic Press, 1977, pp. 104-118.

8. Rolls, B. J. and E. T. Rolls. Thirst. Cambridge: Cambridge University Press, 1982.

9. Rolls, B. J., R. J. Wood and E. T. Rolls. Thirst: the initiation, maintenance, and termination of drinking. Prog Psychobiol Physiol Psychol 9: 263-321, 1980.

10. Rolls, B. J., R. J. Wood, E. T. Rolls, H. Lind, R. Lind and J. Ledingham. Thirst following water deprivation in humans. A m J Physiol 239: R476-R482, 1980.

11. Rolls, B. J., E. A. Rowe and R. C. Turner. Persistent obesity in rats following a period of conumption of a mixed, high energy diet. J Physiol 298: 415-427, 1980.

12. Rolls, B. J., E. T. Rolls, E. A. Rowe and K. Sweeney. Sensory specific satiety in man. Physiol Behav 27: 137-142, 1981.

13. Rolls, B. J., E. A. Rowe, E. T. Rolls, B. Kingston, A. Megson and R. Gunary. Variety in a meal enhances food intake in man. Physiol Behav 26: 215-221, 1981.

14. Rolls, B. J., E. A. Rowe and E. T. Rolls. How sensory properties of foods affect human feeding behavior. Physiol Behav 29: $409-417,1982$.

15. Rolls, E. T. The Brain and Reward. Oxford: Pergamon, 1975.

16. Rolls, E. T. Processing beyond the inferior temporal visual cortex related to feeding, memory, and striatal function. In: Brain Mechanisms of Sensation, edited by Y. Katsuki. R. Norgren and M. Sato. New York: John Wiley, 1981, Ch. 16, pp. 24I-269.

17. Rolls, E. T. Central nervous mechanisms related to feeding and appetite. Br Med Bull 37: 131-134, 1981.

18. Rolls, E. T. Feeding and reward. In: The Neural Basis of Feeding and Reward, edited by D. Novin and B. G. Hoebel. Brunswick, ME: Haer Institute for Electrophysiological Research, 1982.
19. Rolls, E. T. . M. J. Burton and F. Mora. Hypothalamic neuronal responses associated with the sight of food. Brain Res 111: 53-66, 1976.

20. Rolls, E. T. and B. J. Rolls. Activity of neurones in sensory, hypothalamic, and motor areas during feeding in the monkey. In: Food Intake and Chemical Sense's, edited by Y. Katsuki, M. Sato, S. F. Takagi and Y. Oomura. Tokyo: University of Tokyo Press, 1977, pp. 525-549.

21. Rolls, E. T., M. K. Sanghera and A. Roper-Hall. The latency of activation of neurons in the lateral hypothalamus and substantia innominata during feeding in the monkey. Brain Re's 164: 121135,1979

22. Rolls, E. T., M. J. Burton and F. Mora. Neurophysiological analysis of brain-stimulation reward in the monkey. Brain Res 194: $339-357,1980$

23. Rolls, E. T. and B. J. Rolls. Brain mechanisms involved in feeding. In: Psychobiology of Human Food Selection, edited by L. M. Barker. Westport, CT: A.V.I. Publishing Co., 1982, Ch. 3, pp. 33-62.

24. Rolls, E. T., E. Murzi and C. Griffiths. The neurophysiology of feeding. Neurosci $L e^{\prime} t$ 1982, in press.

25. Stunkard, A. J. "Restrained eating": what it is and a new scale to measure it. In: The Body Weight Regulatory System: Normal and Distrubed Mechanisms. edited by L. A. Cioffi, W. P. T. James and T. B. Van Itallie. New York: Raven Press, 1981, pp. $243-251$.

26. Thompson. D. A., H. R. Moskowitz and R. G. Campbell. Effects of body weight and food intake on pleasantness ratings for a sweet stimulus. I App/ Physiol 41: 77-83, 1976.

27. van Duijvenvoorde, P. M., E. A. Rowe and B. J. Rolls. Effects of variety in the diet on food intake and body weight of male and female rats. Int J Obes 1982, in press.

28. Wooley, O. W., S. C. Wooley and R. B. Dunham. Calories and sweet taste: Effects of sucrose preference in the obese and nonobese. Physiol Bchav 9: 765-768. 1972. 